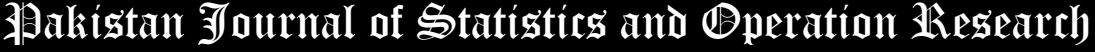

\section{Reliability Estimation of Three Parameters Weibull Distribution based on Particle Swarm Optimization}

\author{
Ghalia Twfeek Basheer ${ }^{1}$, Zakariya Yahya Algamal ${ }^{2 *}$ \\ * Corresponding Author
}

1. Department of Operations Research and Intelligent Techniques, University of Mosul, Mosul, Iraq ghalia2017@yahoo.com, ghalia.tawfeek@uomosul.edu.iq

2. Department of Statistics and Informatics, University of Mosul, Mosul, Iraq, zakariya.algamal@uomosul.edu.iq

\begin{abstract}
The three-parameter Weibull distribution is a continuous distribution widely used in the study of reliability and life data. The estimation of the distribution parameters is an important problem that has received a lot of attention by researchers because of theirs effects in several measurements. In this research, we propose a particle swarm optimization (PSO) to estimate the three-parameter weibull distribution and then to estimate the reliability and hazard functions. The real data results indicate that our proposed estimation method is significantly consistent in estimation compared to the maximum likelihood method. In terms of -log likelihood and mean time to failure (MTTF).
\end{abstract}

Key Words: Reliability, Particle Swarm Optimization, 3-Parameter Weibull Distribution, Maximum Likelihood.

\section{Introduction}

The widespread interest in study of reliability is due to the fast development of the world, especially in the field of technology. The main objective of a reliability study is to provide information as a basis for decision (Rausand and Høyland, 2004). The estimating parameters is the key to the life model, it can predict the life of product accurately in the reliability (Jun et al., 2017). The process of estimating 3-parameter weibull distribution is important because of the difficulty of obtaining the estimated parameters. The estimation process by using the maximum likelihood function requires iterative methods and therefore requires considerable time and effort. In this research, we propose a particle swarm optimization (PSO) to estimate the three-parameter weibull distribution and then to estimate the reliability and hazard functions. The real data results indicate that our proposed estimation method is significantly consistent in estimation compared to the maximum likelihood method.

\section{The weibull distribution}

The weibull distribution introduced by the swedish physicist (Walooddi weibull, 1939). He used it to analyze the breaking strength of materials. Since then, it was widely used in reliability and life testing problems such as the time to failure or life length of a component, measured from some specified time until it fails (Jukić and Marković, 2010) (Walpole et al., 2012).

Let $\mathrm{T}$ a random variable represent time to failure with probability density function $f(t)$, where $f(t)$ is the probability density function of the 3-parameter weibull distribution, that is can be given as (Jukić and Marković, 2010) (Jun et al., 2017).

$$
f(t ; \alpha, \beta, \gamma)=\left\{\begin{array}{cr}
\frac{\alpha}{\beta}\left(\frac{t-\gamma}{\beta}\right)^{\alpha-1} \exp \left[-\left(\frac{t-\gamma}{\beta}\right)^{\alpha}\right] & , t>0 \\
0 & , t \leq 0
\end{array}\right.
$$


Where $\alpha>0$ represents the shape parameter, $\beta>0$ represents the scale parameter, and $\gamma \leq t$ represents the location parameter.

Figure 1 represent the weibull $(\alpha, \beta, \gamma)$ probability density function for various choices of the parameters $\alpha, \beta$ and $\gamma$. Of Figure 1 we note that with different values of the parameters $\alpha, \beta$ and $\gamma$ generate a wide variety of curves. Therefore, the weibull distribution can be made to fit a wide variety of data set. This is the main reason for the use fullness of the weibull distribution (Navidi, 2015).
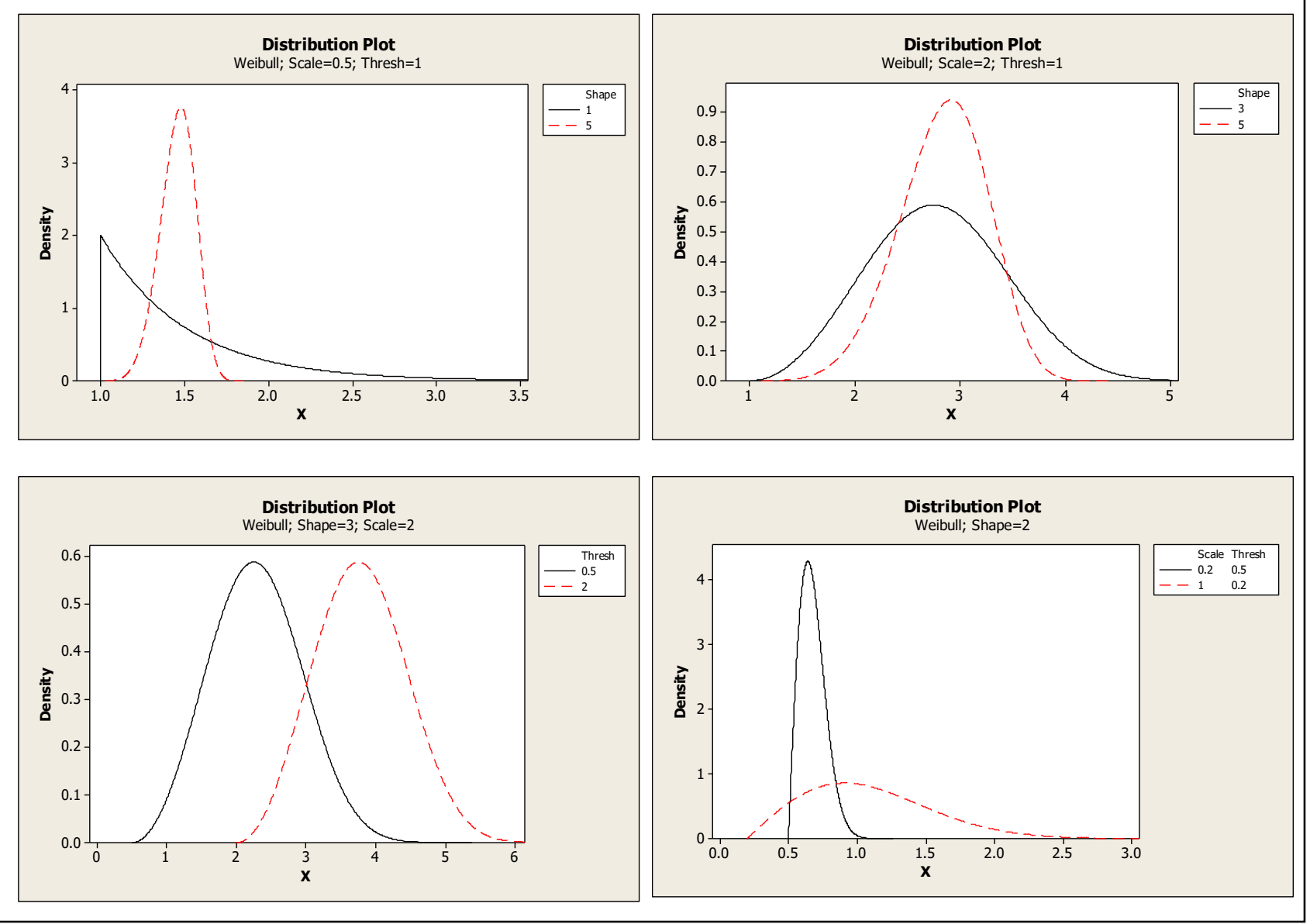

Figure 1: The weibull probability density function for several choices of parameters

The cumulative distribution function for the 3-parameter weibull distribution can be computed by integrating the probability density function

$$
\begin{aligned}
F(t)=P(T \leq t) & =\int_{0}^{t} f(t ; \alpha, \beta, \gamma) d t \\
& =1-e^{-\left[\frac{t-\gamma}{\beta}\right]^{\alpha}}
\end{aligned}
$$

The mean and variance of the 3-parameter weibull distribution are (Muraleedharan, 2013)

$$
\mu=\gamma+\beta \Gamma\left(\frac{\alpha+1}{\alpha}\right)
$$

Where $\mu=E(t)=M T T F$ (mean time to failure is the expected time to failure for system) 


$$
\sigma^{2}=\beta^{2}\left[\Gamma\left(\frac{\alpha+2}{\alpha}\right)-\Gamma^{2}\left(\frac{\alpha+1}{\alpha}\right)\right]
$$

\section{Reliability and failure rate functions}

The reliability function (also called the survival function), is denoted as $R(t)$. It is the probability of successful performance, that is can be write (Haldar and Mahadevan, 2000) (Walpole et al., 2012)

$$
\begin{aligned}
R(t)=P(T>t) & =\int_{t}^{\infty} f(t ; \alpha, \beta, \gamma) d t \\
& =1-F(t) \\
& =e^{-\left[\frac{t-\gamma}{\beta}\right]^{\alpha}}
\end{aligned}
$$

The failure rate (also known as the hazard rate), is denoted as $h(t)$. It is the conditional probability that a component fails in a small time interval from $T=t$ to $T=t+\Delta t$, given that it survived to time $t$, is (Nachlas, 2005) (Walpole et al., 2012)

$$
\begin{aligned}
P(t<T<t+\Delta t \mid T>t) & =\frac{P(t<T<t+\Delta t)}{P(T>t)} \\
& =\frac{F(t+\Delta t)-F(t)}{R(t)}=\frac{\Delta F(t)}{R(t)}
\end{aligned}
$$

By dividing this ratio by $\Delta t$ and finding the limit as $\Delta t \rightarrow 0$, we get the failure rate:

$$
\begin{aligned}
h(t) & =\lim _{\Delta t \rightarrow 0} \frac{P(t<T<t+\Delta t \mid T>t)}{\Delta t} \\
& =\lim _{\Delta t \rightarrow 0} \frac{\Delta F(t)}{\Delta t R(t)}=\frac{f(t)}{R(t)}
\end{aligned}
$$

\section{Maximum likelihood method}

The maximum likelihood method is one of the best methods to estimate parameters. Let $\left(t_{1}, t_{2}, \ldots, t_{n}\right)$ be a random sample of size $\mathrm{n}$ drawn from a population with probability density function $f(t ; \alpha, \beta, \gamma)$, then the likelihood function of this random sample is known as the joint density of the $\mathrm{n}$ a random variables, which can be written as (Jun et al., 2017)

$$
L\left(t_{1}, t_{2}, \ldots, t_{n} ; \alpha, \beta, \gamma\right)=\prod_{i=1}^{n} \frac{\alpha}{\beta}\left(\frac{t-\gamma}{\beta}\right)^{\alpha-1} \exp \left[-\left(\frac{t-\gamma}{\beta}\right)^{\alpha}\right]
$$

Taking the logarithms of both sides equation (6) yields:

$$
\ln L=n \ln (\alpha)-n \ln (\beta)+(\alpha-1) \sum_{i=1}^{n} \ln \left(\frac{t_{i}-\gamma}{\beta}\right)-\sum_{i=1}^{n}\left(\frac{t_{i}-\gamma}{\beta}\right)^{\alpha}
$$

And taking the partial derivatives of equation (7) for , $\beta$ and $\gamma$, we obtain the following estimated equations:

$$
\frac{\partial \ln L}{\partial \alpha}=\frac{n}{\alpha}+\sum_{i=1}^{n} \ln \left(\frac{t_{i}-\gamma}{\beta}\right)-\sum_{i=1}^{n}\left(\frac{t_{i}-\gamma}{\beta}\right)^{\alpha} \ln \left(\frac{t_{i}-\gamma}{\beta}\right)=0
$$




$$
\begin{aligned}
& \frac{\partial \ln L}{\partial \beta}=-\frac{n \alpha}{\beta}+\frac{\alpha \sum_{i=1}^{n}\left(t_{i}-\gamma\right)^{\alpha}}{\beta^{\alpha+1}}=0 \\
& \frac{\partial \ln L}{\partial \gamma}=-(\alpha-1) \sum_{i=1}^{n} \frac{1}{t_{i}-\gamma}+\frac{\alpha}{\beta} \sum_{i=1}^{n}\left(t_{i}-\gamma\right)^{\beta-1}=0
\end{aligned}
$$

We note that the above three equations are nonlinear, so the newton raphson method is used to obtain the maximum likelihood estimated of the parameters.

\section{Particle swarm optimization}

Swarm intelligence is a branch of artificial intelligence which that used widely for solving optimizition problems that took its inspiration from the biological (Parsopoulos and Vrahatis, 2010) (Premalatha and Natarajan, 2009).

In (1995) Kenned and Eberhart proposed a stochastic optimization algorithm called particle swarm optimization (PSO). The PSO algorithm is very simple, easily and its fast convergence compared with the other optimization algorithms (Premalatha and Natarajan, 2009) (Rao, 2009).

In PSO suppose that the search space is D-dimensional an $\mathrm{N}$ particles in swarm. Each particle $i$ has a position $x_{i}=$ $\left(x_{i 1}, x_{i 2}, \cdots \cdots, x_{i D}\right)$ in the search space. Each particle $i$ moves with in search space by velocity denoted as $v_{i}=\left(v_{i 1}, v_{i 2}, \cdots \cdots\right.$ ,$\left.v_{i D}\right)$, where $i=1,2, \cdots \cdot, N$. The new velocity and position vectors in each iteration are according to the following equations (Handoyo et al., 2017)

$$
\begin{aligned}
& v_{i}(t)=w v_{i}(t-1)+c_{1} r_{1}\left[P_{\text {best }, i}-x_{i}(t-1)\right]+c_{2} r_{2}\left[G_{\text {best }}-x_{i}(t-1)\right] \\
& x_{i}(t)=x_{i}(t-1)+v_{i}(t), \quad i=1,2, \cdots \cdots \cdots, N
\end{aligned}
$$

Where $w$ is the intertia weight, $c_{1}, c_{2}$ are the congnitive and social learning rates respectively, it is usually assumed to be 2 , $r_{1}, r_{2}$ are the random numbers in the range 0 and 1 .

The value of inertia weight decreases linearly with the iteration number has been used:

$$
w(t)=w_{\max }-\left(\frac{w_{\max }-w_{\min }}{t_{\max }}\right) t
$$

Where $w_{\max }$ and $w_{\min }$ represented the initial and final values of the inertia weight, the values of $w_{\max }$ and $w_{\min }$ are usually assumed to be 0.9 and 0.4 respectively, $t_{\max }$ is the maximum number of iterations (Rao, 2009).

\section{The proposed method:}

Consider the equation (13) is the fitness function of the particle swarm optimization algorithm

$$
-\ln L=-\left[n \ln (\alpha)-n \ln (\beta)+(\alpha-1) \sum_{i=1}^{n} \ln \left(\frac{t_{i}-\gamma}{\beta}\right)-\sum_{i=1}^{n}\left(\frac{t_{i}-\gamma}{\beta}\right)^{\alpha}\right]
$$

Where the parameters $\alpha, \beta$ and $\gamma$ are the search variables. The aim is to find the values of parameters $\alpha, \beta$ and $\gamma$ such that the function $-\ln L$ is a minimum.

Let $\theta_{i}(t)$ is the position vector of particle $i$ in the multidimensional search space in step $(\mathrm{t})$, where $\theta_{i}(t)=$ $\left(\begin{array}{lll}\alpha & \beta & \gamma\end{array}\right)$, then the procedures of the solution of the proposed method are illustrated in the following steps:

1. Choose the size of the swarm $\mathrm{N}=50$.

2. Generate the initial population of $\theta$ using lower and upper bounds of the parameters values, $\theta^{L}$ and $\theta^{U}$, randomly using the following equation: 


$$
\theta_{i}(0)=\theta^{L}+\operatorname{rand}()\left(\theta^{U}-\theta^{L}\right) \quad, i=1,2, \cdots \cdots \cdots, N
$$

where $\theta^{L}=\left(\begin{array}{lll}\alpha^{L} & \beta^{L} & \gamma^{L}\end{array}\right)$ the lower bound of parameters $\left(\begin{array}{lll}\alpha \beta & \gamma\end{array}\right)$, for data1 and data2 $\theta^{L}=\left(\begin{array}{lll}0 & 0 & 0\end{array}\right), \theta^{U}=$ $\left(\begin{array}{lll}\alpha^{U} & \beta^{U} & \gamma^{U}\end{array}\right)$ the upper bound of parameters $\left(\begin{array}{lll}\alpha & \beta\end{array}\right)$, for data1 $\theta^{U}=\left(\begin{array}{lll}200 & 200 & 2.5\end{array}\right)$ and data2 $\theta^{U}=$

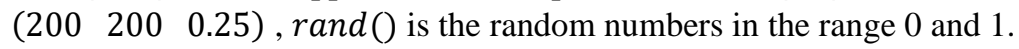

And compute the fitness function values corresponding to the each particles as , $-\ln L\left(\theta_{1}(0)\right),-\ln L\left(\theta_{2}(0)\right)$, $\cdot,-\ln L\left(\theta_{N}(0)\right)$.

3. Compute the velocity of each particle. The initial velocities of all particles are assumed to be zero. Set the iteration number as $\mathrm{t}=1$.

4. Find two important parameters:

a. The historical best value of $\theta_{i}(t), P_{\text {best }, i}$, with the smallest value of the fitness function, $-\ln L\left(\theta_{i}(t)\right)$, encountered by particle $i$ in all the previous iterations.

The historical best value of $\theta_{i}(t), G_{\text {best }}$, with the smallest value of the fitness function, $-\ln L\left(\theta_{i}(t)\right)$, encountered in all the previous iterations by any of the $\mathrm{N}$ particles.

b. Update the velocities of the particles

$$
v_{i}(t)=w v_{i}(t-1)+c_{1} r_{1}\left[P_{\text {best }, i}-\theta_{i}(t-1)\right]+c_{2} r_{2}\left[G_{\text {best }}-\theta_{i}(t-1)\right]
$$

5. Update the position of each particle by:

$$
\theta_{i}(t)=\theta_{i}(t-1)+v_{i}(t), \quad i=1,2, \cdots \cdots \cdots, N
$$

and find the fitness function values corresponding to the particles as, $-\ln L\left(\theta_{1}(t)\right),-\ln L\left(\theta_{2}(t)\right), \cdots \cdots \cdots \cdots \cdots$ ,$-\ln L\left(\theta_{N}(t)\right)$.

6. Check the convergence if the positions of all particles converge to the same set of valued stop, otherwise go to step 4, updating the iteration number, $\mathrm{t}=\mathrm{t}+1$.

\section{Real Data Results}

In this section, we taken two data sets from (Saffawy and Al-Jammal, 2006), it represent the operating times for two different machine. In order to test the fitting of the both data sets for 3-parameter weibull distribution, the kolmogorov-smirnov test was used with values 0.15554 and 0.13448 and the corresponding P-values are 0.43999 and 0.54466 respectively. Therefore it refers that 3-parameter weibull distribution can be fitted to both data sets.

For the purpose of estimating the reliability function of the 3-parameter weibull distribution, the methods described in this paper were used. In order to obtain the best estimate, the log likelihood and mean time to failure (MTTF) was used to compare between methods. Table 1 and Table 2 are represents comparison between MLE and PSO for data1 and data2 respectively.

Table 1: Data 1 sample size 29

\begin{tabular}{cccc}
\hline Parameters & ML & PSO & Best \\
\hline$\hat{\alpha}$ & 0.72694 & 0.7338 & \\
$\hat{\beta}$ & 94.181 & 107.5100 & \\
$\hat{\gamma}$ & 2.49 & 2.49 & PSO \\
-log likelihood & 167.0152 & 166.8843 & \\
MTTF & 117.6497 & 132.8754 & \\
\hline
\end{tabular}


Table 2: Data 2 sample size 33

\begin{tabular}{cccc}
\hline Parameters & ML & PSO & Best \\
\hline$\hat{\alpha}$ & 0.93498 & 0.96 & \\
$\hat{\beta}$ & 93.64 & 96.3216 & \\
$\hat{\gamma}$ & 0.24 & 0.24 & PSO \\
-log likelihood & 184.2477 & 184.2252 & \\
MTTF & 96.8224 & 98.3279 & \\
\hline
\end{tabular}

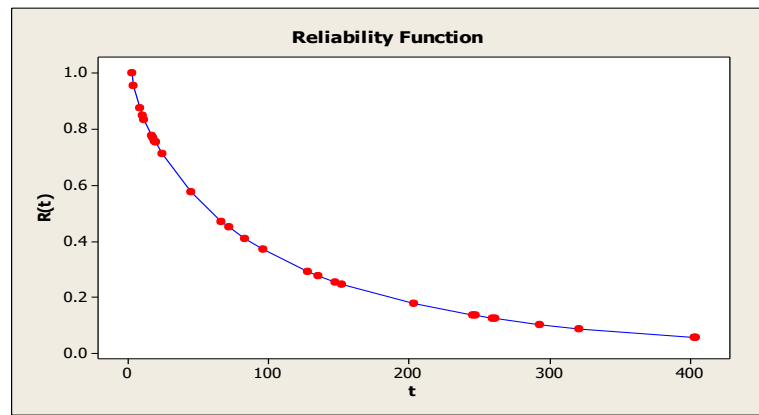

a) Reliability function for MLE of datal

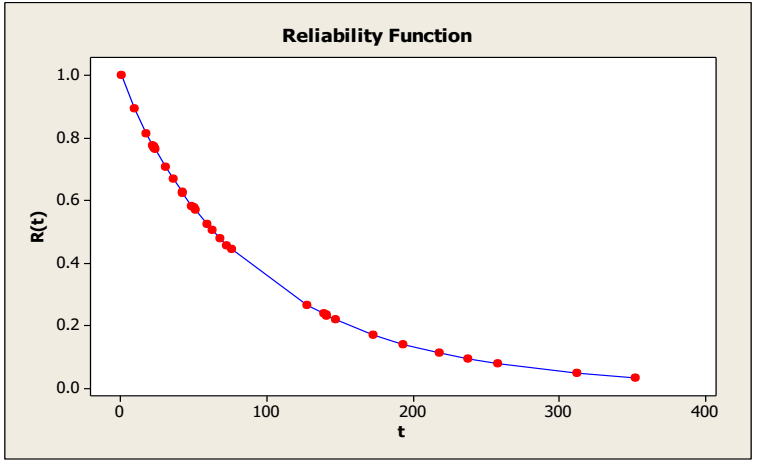

c) Reliability function for MLE of data2

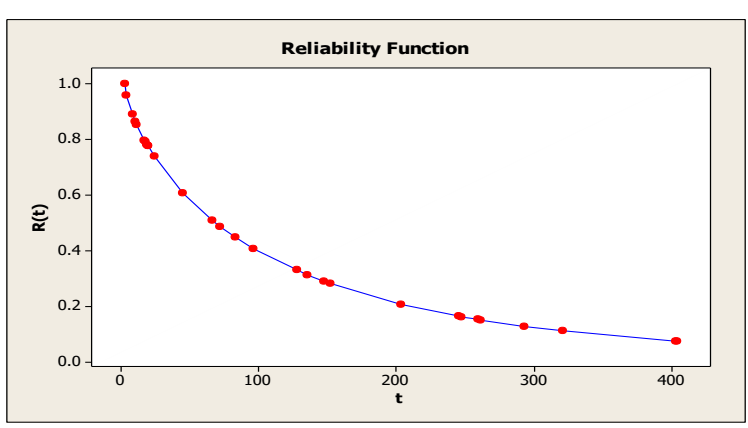

b) Reliability function for PSO of data1

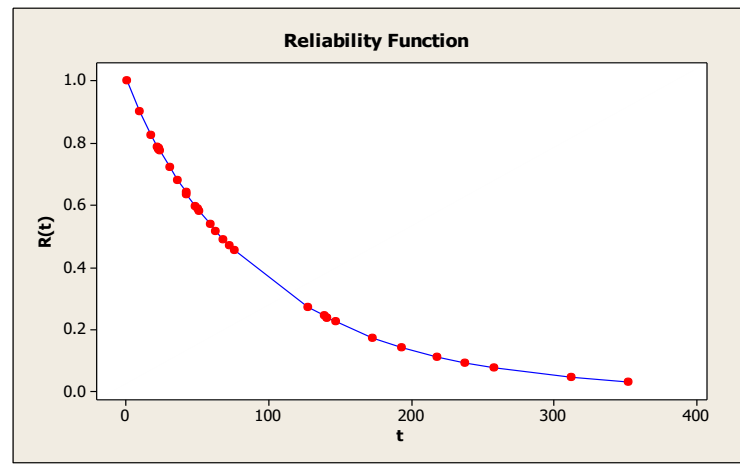

d) Reliability function for PSO of data2

Figure 2: The reliability function using ML and PSO estimators 


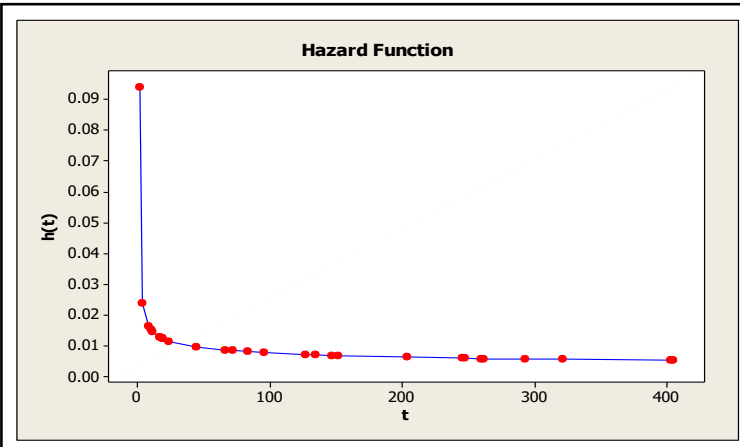

a) Hazard function for MLE of data1

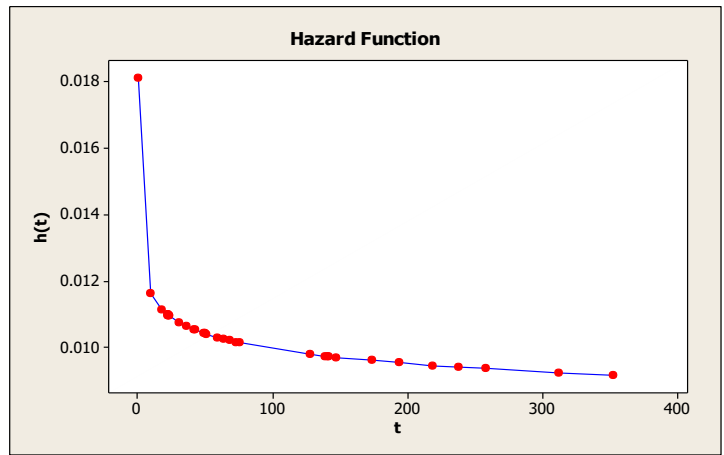

c) Hazard function for MLE of data2

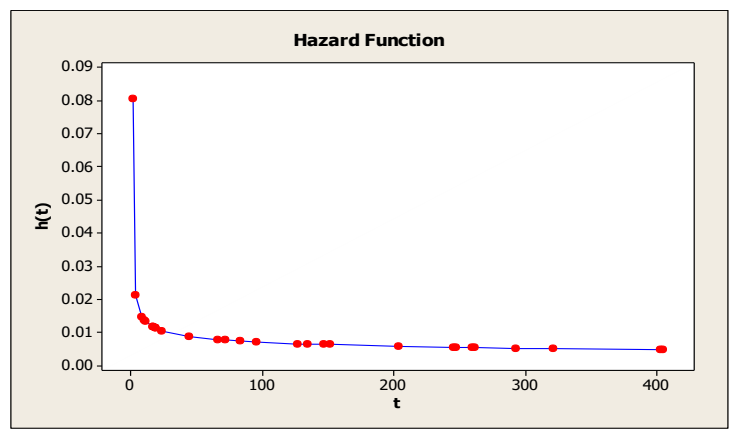

b) Hazard function for PSO of data1

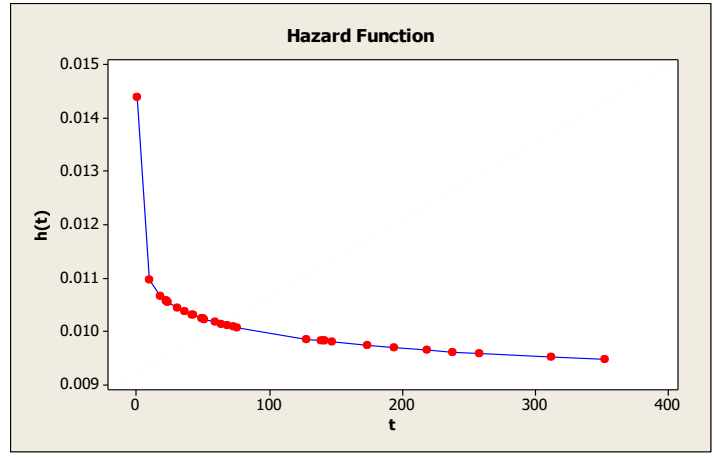

d) Hazard function for PSO of data2

Figure 3: The Hazard function using ML and PSO estimators

\section{Conclusion}

Estimating parameters of 3-parameter weibull distribution is an important factor in reliability. The estimation of weibull distribution parameters with three parameter using traditional techniques such as maximum likelihood function is difficult because it is nonlinear functions. In this paper we proposed method based on swarm intelligence, PSO, to estimate these parameters. Comparison with the ML the proposed method showed higher accuracy and efficiency in the estimation, and don't need compute the partial derivatives as in the ML.

\section{References}

1. Jukić D., Marković D, (2010). On nonlinear weighted total least squares parameter estimation problem for the three-parameter weibull density. Applied Mathematical Modelling, 34, 1839-1848.

2. Jun W.C, Xin L, Xin C., (2017). Parameter Evaluation of 3-parameter weibull Distribution based on Adaptive Genetic Algorithm. 2 $2^{\text {nd }}$ International Conference on Machinery, Electronics and Control Simulation, Advances in Engineering Research, volume 138.

3. Haldar A., Mahadevan S., (2000). Probability, Reliability and Statistical Methods in Engineering Design. John Wiley and Sons, Inc. 
4. Handoyo S., Efendi A., Jie F., Widodo A., (2017). Implementation of Particle Swarm Optimization (PSO) Algorithm for Estimating Parameter of ARMA Model via Maximum Likelihood Method . Far East of Mathematical Sciences (FJMS), Volume 102, Number 7, pages 1337-1363.

5. Muraleedharan G., (2013). Characteristic and Moment Generating Functions of Three Parameter Weibull Distribution-an Independent Approach. Research Journal of Mathematical and Statistical Sciences, Vol.1(8), 25-27.

6. Nachlas J.A., (2005). Reliability Engineering Probabilistic Models and Maintenance Methods. Taylor \& Francis Group.

7. Navidi W., (2015). Statistics for Engineers and Scientists.Mc Graw-Hill Education, Fourth edition.

8. Parsopoulos K.E., Vrahatis M. N., (2010). Particle Swarm Optimization and Intelligence Advances and Applications. United States by America, IGI Global.

9. Premalatha K., Natarajan A. M., (2009). Hybrid PSO and GA for global maximization. Int. J. Open problems compt. Math.,Vol. 2, No. 4, p.p. 597-608.

10. Saffawy S.Y., Algmal Z.Y. , (2006). The Use of Maximum Likelihood and Kaplan-Meir method to Estimate the Reliability Function An Application on Babylon Tires Factory. AL-Rafidain Development Journal, Vol. 82, No. 28.

11. Rao S.S., (2009). Engineering Optimization Theory and Practice. John Wiley and Sons, Inc. 4th ed.

12. Rausand M. \& Høyland A.,(2004). System Reliability Theory. John Wiley and Sons, Inc. 2th ed.

13. Walpole R.E., Myers R. H., Myers S.L. and Ye K., (2012). Probability and statistics for Engineers and Scientists. Pearson Education, Inc. 